\title{
Balancing Quality and Performance of Task Distribution in Workflow Based on Resource Aptitude
}

\author{
Renê Rodrigues Veloso ${ }^{1}$ and Autran Macêdo ${ }^{2}$ \\ ${ }^{1}$ FACIT - College of Science and Technology, Department of Computer Engineering, \\ Brazil \\ ${ }^{2}$ Federal University of Uberlandia, School of Computing, Brazil
}

\begin{abstract}
The distribution of tasks in a workflow system, in general, does not consider resource aptitude. Resources that share the same role in a workflow can have different degrees of aptitude to a task. This work proposes an extension to task distribution strategies and presents the use of this extension in the selection of resources to tasks. The experiments presented here demonstrate the effectiveness of the extension. A comparison with traditional strategies has shown gains in terms of time and quality of tasks executed.
\end{abstract}

Keywords: Workflow, Performance, Quality, Aptitude, Task distribution.

\section{Introduction}

Task distribution to resources is one of the critical functions of Workflow Management Systems (WfMS) 12 and is directly related to time of completion and quality of the execution process 345].

Task distribution mechanisms based on aptitude can be used in the prevention of workflow bottlenecks. Being resource oriented, theses mechanisms are able to use the information found in the capacities and abilities of available resources. With this, it is possible to safeguard against inadequate and/or busy resources receiving tasks, therefore avoiding the occurance of bottlenecks.

There are proposals of hybrid approaches between Push and Pull [26|7/8 9]. In this case, resources have individual queues of tasks that are filled by an heuristic that moves tasks of a shared queue to individual queues. This heuristic is batchbased with the purpose of minimizing flowtime, i.e., the difference between the beginning and the end of a task. It create groups of tasks based on their process time. However, none of this related research is worried about maximizing quality, maintaining a satisfactory execution time of the business process.

The research of Kumar et al. 4], proposes distribution mechanisms that, beyond considering the aptitude of resources, tries to balance quality and performance through rules that restricts the visibility of resources to tasks. However, as the mechanisms there proposed are strongly based on the Pull mechanism, the 
choice of tasks are made by resources, which can facilitate the occurance of bottlenecks. Apart from the Kumar et al. research, the mechanisms proposed and explored in this paper are implemented as Push mechanisms, where the system chooses the resources at the moment of distribution. Permitting the system to totally guide the distribution can improve the quality and performance goals of balancing, because these goals are controlled and leveled according to resource availability and aptitude to each delivered task.

\subsection{Solution Approach}

Resource aptitude can be surveyed by means of metrics, which indicate the degree of adequacy of resources to the completion of tasks (4]). In other words, the degree of adequacy measures the level of competence of resources for the realization of the tasks of the workflow. keeping to the context of this paper, resources with high degree of adequacy to a given task has greater aptitude for the execution of the tasks. So the fact that these resources perform tasks more efficiently than those less adequate to the tasks is to be expected.

The metrics of allocation are defined as a systematic solution to dynamically create equilibrium between quality and performance. This equilibrium is obtained through the construction of a model directionally toward better distribution. In this way, the systems should know how to choose the most apt resources to do tasks in any given process which one desires to automate.

Therefore, the purpose of this paper has two approaches: (i) to develop efficient task distribution mechanisms to apt resources, which may be applied to various types of businesses. (ii) to make a comparative study of existing traditional mechanisms. These implemented mechanisms should be able to facilitated gains in work execution efficiency of workflow systems, taking in to consideration the resource aptitude to the task and the workload already allocated. To realize this goal, section 2 describes the metrics used by the distribution mechanisms to allocate resources and also to measure the quality of the execution processes, as well as their respective parameters. The section 3 shows mechanisms based on resource aptitude and describes the proposal Sel-push e Sel-push-10, to balance both quality and performance. In the section 4, a computer experiment to compare the performance and quality of mechanisms is detailed, and the results analyzed considering various kinds of problems. In the end, the conclusion is commented on, followed by the bibliographical references used in this paper.

\section{Allocation Metrics}

The allocation metric presented in 4 was originally propose as criteria of commitment between quality and performance for a workflow system. This proposal relates resources and tasks by means of parameters such as adequacy and availability. 1

\footnotetext{
${ }^{1}$ Other parameters are defined by Kumar et al. as being urgent to the execution of tasks and degree of violation of restriction in the modeled process. These were disconsidered in this paper (the tasks are equally urgent and do not exist restrictions).
} 
The parameters are combined in a metric which determines the allocation of the task to a resource. This metric called a absolute allocation factor or, simply, abs_alloc, is defined by:

$$
\operatorname{abs\_ alloc}(w, r s)=\operatorname{adequacy}(w, r s) \times \operatorname{availability}(r s) .
$$

The adequacy parameter is about the aptitude of a resource in relation to a given task. This parameter is given by the function $\operatorname{adequacy}(w, r s) \in[0,1]$, in $w$ is a workflow task and $r s$ is a workflow resource. The lower the adequacy value, the lower aptitude $r s$ possesses to execute $w$.

Availability indicates how available a resource is to perform a task. This parameter is expressed by the function availability $(r s) \in[0,1]$. Values lower than 1 indicates that the resource in question is already allocated to some task. A value equal to zero indicates that the resource is unavailable.

The metric abs_alloc depends only on a task and a resource isolatedly. This metric assumes a value between 0 and 1 , which is an absolute measure of adequacy to a resource to perform a task immediately. The higher the value of abs_alloc of a resource, more adequate it will be to execute the task. 2

\section{Distribution Mechanisms Based on Aptitude}

The distribution of tasks in workflow systems, in general, occurs through the Push and Pull mechanisms [10. However, neither of these mechanisms considers the resource aptitude to the tasks.

In this sense, Kumar et al. 4 defines three mechanisms based on Pull. These mechanisms are practically based on the definited adequacy thresholds that increase or decrease the amount and the quality of the involved resources. These thresholds are as follows:

- Typical: one task $w$ is visible to resource $r s$ if, and only if, abs_alloc(w, rs) exceeds a value 0.5 . This represents a typical resource.

- Receptive: one task $w$ is visible to resource $r s$ if, and only if, abs_alloc(w, rs) exceeds a lesser value than the typical one, e.g., 0.4. This indicates a more receptive resource.

- Selective: one task $w$ is visible to a resource $r s$ if, and only if, abs_alloc(w, rs) exceeds a value higher than the typical one, e.g., 0.6. This indicates a more selective resource than the typical resource.

The proposal of this paper involves an extension to the mechanisms of distribution cited previously. Also taking as base the values of absolute allocation, the differential of this extension is in the form which the tasks are delivered to the resources. Before the resources receive the tasks, their availability values are verified. When receiving tasks, the resources are less available in the context of the system. In this way, a resource that has a high workload will not have to receive more tasks, these being repassed to other resources that are apt and have availability to execute them. Based on Push, the extension is implemented as two other mechanisms are: the Sel-Push and the Sel-Push-10.

\footnotetext{
${ }^{2}$ In the equation 1 the disconsidered parameters both assume a value equal to one.
} 


\subsection{The Sel-Push Distribution Mechanism}

In a simplified manner, the distribution mechanism Sel-Push is called to action whenever the task queue (ready) is not empty and continues until the tasks of the queue are allocated, or until the resources are no longer available. In this case, the mechanism goes into waiting mode until some resource becomes available. An choice of the resource with greatest abs_alloc occurs guided by a dynamic threshold. This means, a resource which is initially found inside a margin of selective threshold, depending on how much this resource is being attributed tasks, its value of abs_alloc suffers a decrease, going to the typical threshold and after that, to the receptive threshold. The Sel-Push verifies beforehand if the resources most apt to the task are selective, and if they are not, others are sought for within the boundaries of inferior thresholds.

Of similar working to the mechanism Sel-Push, the Sel-Push-10 mechanism tries to pass tasks to the most apt resources. Therefore, besides choosing the resource with greater abs_alloc, it also considers the neighborhood of this resource. The neighborhood of a resource are all the resources within a certain numerical distance from adequacy value of this resource. In the case of Sel-Push-10 the neighborhood is within a radius of $10 \%$ of abs_alloc of the most adequate resource.

\section{Computational Study}

Considering the little information available about other solutions to the problem of task distribution in workflow systems, an analytical evaluation of all other existing mechanisms go far beyond the scope of this paper. Therefore, a computational study was done to compare the performance and quality of seven mechanisms, i.e., Push, Pull, Selective, Typical, Receptive, Sel-Push and Sel-Push-10. It is importante to stress that the mechanism Push in this study, attributes the tasks to always the same resource with the greatest adequation value. In this section, the procedures used to simulate and presents the statistics based on the results of simulation are described.

\subsection{Workload Generation}

To control the workload of the computational study, a model proposed by Zeng and Zhao 2 is used. The idea of this model is centralized mainly in the evaluation of quality and performance of the mechanisms under study of varying business work environments. The parameters which characterize these scenarios of environments and their adjustments examined in this study are presented in the Table 1 Only a small number of resources were used (3 and 6), which is sufficient for the simulation of a organizational workflow environment.

In the model, the medium system load measures the number of tasks that arrive. This number is related to the capacity of the resource. Therefore, the control of the dynamic generation of tasks and the process used by Zeng and Zhao is detailed as following. With the processing time of each task fixed in 
Table 1. Simulation Parameters

\begin{tabular}{lc}
\hline Resource number & \multicolumn{1}{c}{3 and 6 } \\
Medium system load & 0.2 (low), 0.6(medium), 1.0(high) \\
Variability of tasks & 20 (low), 80(medium), 140(high) \\
Variability of resources & 20 (low), 80(medium), 140(high) \\
\hline
\end{tabular}

10 units of time, the medium capacity of a resource for each unit time is $1 / 10$. Assuming that $n$ resources are presented in the system, the total capacity of resources per unit of time is $n / 10$. In this way, a medium system workload $l$, the number of tasks which arrive during each unit of time follows a Poisson distribution with $\lambda=l \times n / 10$.

Two other parameters of the table, the variability of tasks and of resources control the time adjustment of processing of the task $p_{i j}$. In this study, this time is generated in two steps:

- Step 1. For the task $j$, the average processing time $\overline{p_{j}}$ is generated based on the normal distribution with average of 10 (average time of each task) and standard deviation varTask. Therefore, $\overline{p_{j}} \approx N\left(10,(10 \times \text { varTask })^{2}\right)$. For example, considering a process with three tasks and a low variability of tasks, we have a processing time varying between 8 and 12 units.

- Step 2. The processing time of task $j$ by resource $i$ expressed by $p_{i j}$, is obtained after the average $\overline{p_{j}}$. So varTask, the resource variability varResource indicates the percentage standard deviation of the individual processing of a task. Therefore, $p_{i j}$ is generated from a normal distribution where: $p_{i j} \approx N\left(\overline{p_{j}},\left(\overline{p_{j}} \times \operatorname{varResource}\right)^{2}\right)$.

All of the values of average time generated by the model were rounded out to whole numbers and negative values to the value 1 .

\subsection{Execution of the Simulation}

The Table 1 represents a total of $2 \times 3 \times 3 \times 3=54$ experimental conditions. For each condition, 20 instances of problems had been generated randomly and then the seven mechanisms were applied to the these instances. In the total, 7560 instances had been simulated. For the proposed study, a simulator able to execute the instances under any one of the described mechanisms of distribution was implemented [11]. Writing in Java(TM) language, the program is based on the analysis of charts and concepts of the Scheduling area $([12])$, to compute the times of execution and the allocations of the tasks for the resources.

Among the data that is generated by the simulator after the execution of the instances is the total execution time of the processes and the value of quality, resultant of the attributions made for the distribution mechanisms. This data was collected and is analyzed later in order to make possible the identification of the mechanism with greater commitment to performance and quality. For reasons of space, only the results based on the execution average of the 3 system load types, i.e., low load (20\%), average (60\%) and high (100\%) will be presented. In 
the same way, each data point on the result graphs is the average of 20 instances with the same system load and processing time variation of only 3 different resources concurring for each task.

In the simulated model, two independent parameters exist, i.e., task variability and resource variability, each one with three different possible values (low, medium and high) resulting in nine different combinations. However, only four of the possible representative combinations are shown. Thus (varTask, varResource $) \in\{($ Low, Low $),($ Low, High $),($ Medium, Medium $),($ High, High $)\}$ is seen to be enough for the intention of the paper. The target process of the simulations contains three tasks. Initially all of the resources possess high availability. The adequation values of each resource are empiric and generated randomly.

\subsection{Total Execution Time and Quality of the Processes}

The behavior of seven mechanisms was evaluated in terms of total execution time and quality of the processes. It is also observed that the execution times and quality of the processes of the Push mechanism surpasses the others mechanisms in all variations of task and resource. The Selective mechanism, with similar behavior, also has high time and quality indices. These results occur due to the fact that these mechanisms are very restrictive, delivering tasks always to a single resource or to a small group of resources. However, in consequence, waiting queues occur for more adequate resources, accumulating tasks and increasing the completion time of processes. The mechanisms Pull and Typical result in less time and quality in execution because they are very liberal, i.e., they make it difficult to accumulate tasks because they involve a greater number of resources. The mechanisms based on Sel-push, combine the characteristics of both the liberal and restrictive mechanisms distributing tasks better.

Table 2. Comparison of mechanisms for execution time and quality

\begin{tabular}{ccc}
\hline & Sel-Push Sel-push-10 \\
\hline Push & $-/+$ & $-/+$ \\
Pull & $+/-$ & $+/-$ \\
Selective & $-/=$ & $-/+$ \\
Typical & $=/-$ & $=/-$ \\
Receptive & $+/-$ & $+/-$ \\
Sel-push & & $-/+$ \\
\hline
\end{tabular}

The observations shown previously are confirmed by the execution of some statistical tests. For example, the Table 2 contains comparisons proceeding from a statistical test called pairwise t-test, used to see if the results of each mechanism can be considered statistically equal to each other. In this table there are three symbols: the symbol "+", the symbol "-" and the symbol "=". These symbols, in each column, are in the format symbol/symbol representing time/quality. The symbol "+" means that the mechanism that is in the row on the table is statistically better than the mechanism which is in its respective column, with 
$95 \%$ of confidence. The symbol "-", contrary to the symbol "+", means that the mechanism which is in the row is statistically worse than the mechanism in the column. In the case of the symbol "=", also demonstrating the same $95 \%$ of confidence, confirms that there is no significant difference between the corresponding row and column of the table.

It is observed that through testing, that the mechanisms behave in a constant manner regarding task variation, resources and system load. The more restrictive mechanisms elevate the time necessary to conclude processes due to task accumulation. Although, for the same reason, the mechanisms raise the average quality of executed process. On the other hand, while the more liberal mechanisms spend less time in the execution of processes because they have more resources receiving tasks, at the same rate favor the decreasing service quality. Therefore, the mechanisms Sel-Push and Sel-Push-10, englobe the best of these two worlds, because besides guaranteeing a average time of execution of the processes similar to the processes dealt with by the liberal mechanisms, they keep a high level of quality, equaling the more restrictive mechanisms.

(a)

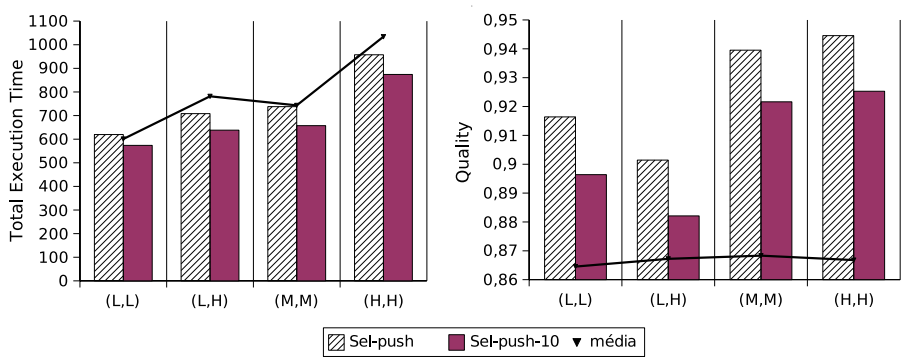

Fig. 1. Average Curve of (a) execution time and (b) quality of processes

The graph of the Figure 1 resumes the behavior of the Sel-Push and SelPush-10 mechanisms in relation to the average curves of execution time and quality of executed processes with Push, Pull, Selective, Typical and Receptive mechanisms. Conforming to the graph, the mechanism Sel-push has an average behavior in relation to execution time and quality better than the other analyzed mechanisms. This betterment becomes more evident as the variation of processing time of tasks increases.

\section{Conclusions}

The task distribution, besides being a key toward dynamically attributing task which arrive individually for resources, directly affects the service quality and the resource productivity. It also is an integral part of decisions of the strategical level of the organizations [2]. 
Two task distribution mechanisms were proposed. Their performance was studied by simulation. The results were compared to other mechanisms which reflect the current practice in workflow. The study done concentrated on analysis of two factors of performance: quality and execution time of work. The impact of variation of individual processing time of tasks, the number of resources involved and the system load of these performance factors were evaluated under the seven evaluated mechanisms.

It was observed that the proposed based on Push mechanisms obtained effective gains in average quality and execution time in simulated scenarios.

\section{References}

1. Stohr, E.A., Zhao, J.L.: Workflow automation: Overview and research issues. Inform. Systems Frontiers 3 (2001) 281-296

2. Zeng, D.D., Zhao, J.L.: Effective role resolution in workflow management. INFORMS journal on computing 17(3) (2005) 374-387 ISSN: 1091-9856.

3. Governatori, G., Rotolo, A., Sadiq, S.: A model of dynamic resource allocation in workflow systems. In: XV Australasian Database Conference. Volume 27., Dunedin, New Zealand (2004)

4. Kumar, A., van der Aalst, W.M., Verbeek, E.M.: Dynamic work distribution in workflow management systems: How to balance quality and performance? Journal of Management Information Systems 18(3) (2001) 157-193

5. Momotko, M., Subieta, K.: Dynamic changes in workflow participant assignment. In: ADBIS Research Communications. Volume 2., Bratislava, Slovakia, Slovak University of Technology, Bratislava (setembro 2002) 175-184

6. Alt, R., Klein, S., Kuhn, C.: Service task allocation as an internal market. In: Proc. of the Second European Conf. on Information Systems. (1994) 424-432

7. Harker, P.T., Ungar, L.H.: A market-based approach to workflow automation

8. Tan, J.C., Harker, P.T.: Designing workflow coordination: centralized versus market-based mechanisms. Technical report, Department of Systems Engineering, University of Pennsylvania, Philadelphia (1997)

9. Shen, M., Tzen, G.H., Liu, D.R.: Multi-criteria task assignment in workflow management systems. In: Proc. Thristysixth Hawaii Internat. Conf. System Sci., Big Island, Hawaii 202

10. van der Aalst, W.M.P., van Hee, K.M.: Workflow Management: Models, Methods, and Systems. MIT Press (2002)

11. Veloso, R.R., Macêdo, A.: Lambari: Simple workflow test environment. Technical report, Department of Computer Science, Federal University of Uberlandia, MG, Brazil (2005)

12. Brucker, P.: Scheduling Algorithms. Springer-Verlag New York, Inc., Secaucus, NJ, USA (1998) 